\title{
Preface to the 30th Anniversary Special Issue of Acta Mechanica Solida Sinica
}

\author{
Daining Fang ${ }^{1 \star}$ \\ $\left({ }^{1}\right.$ Beijing Institute of Technology, Beijing 100081, China)
}

\begin{abstract}
Received 18 August 2018; revision received 21 August 2018; Accepted 23 August 2018; published online 25 September 2018

(c) The Chinese Society of Theoretical and Applied Mechanics 2018
\end{abstract}

Together with the effort of the Chinese Society of Theoretical and Applied Mechanics and the Editorial Board members, the international journal Acta Mechanica Solida Sinica (AMSS) was founded in the spring of 1988. Thanks to the continued support from our authors and readers over the past 30 years, AMSS has become an important platform for documenting the state-of-the-art development and sharing innovative ideas in the theories and engineering applications of Solid Mechanics. Since 1996, the published papers of $A M S S$ have been indexed by the Science Citation Index Expanded (SCIE) database. The journal's influence has been enhanced with a continuous increase in its impact factor. It is remarkable to note that the journal has been listed in "the Highest International Impact Academic Journals of China" for 5 years in a row.

Our appreciation also goes to all our previous editors-in-chief and Editorial Board members. They contributed in so many ways that to say the journal of $A M S S$ couldn't have made such progress without them would be a gross understatement. Dating back to 30 years ago, Prof. Qinghua Du, a pioneer of Aeronautic and Astronautic Material Engineering in China, became the first Editor-in-Chief of AMSS at the age of almost 70. To make Acta Mechanica Solida Sinica known to the world, he introduced the research of Solid Mechanics conducted in China by giving plenary talks at international conferences and symposia, invited well-recognized researchers to serve on the Editorial Board and International Advisory Editorial Board of $A M S S$, and gained assistance and support worldwide for the journal by paying visits abroad. After established, the journal of $A M S S$ was published quarterly for 20 years. Since 2008 , to accommodate the increasing submissions and shrink the turnaround time, it has grown from four to six issues annually.

Numerous efforts have been devoted to making $A M S S$ an international academic journal with great impact, a premier platform for the exhibition and communication of Solid Mechanics and pertinent interdisciplinary research advancements, and a medium to manifest the crucial role of Solid Mechanics in science and technology development. To achieve the criteria for a high-quality journal, we have been working hard to maintain the strict scientific standard and resonance in the international community, and doing our best to serve every individual author with prompt, constructive and valuable responses. In 2018, the average turnaround time for manuscripts submitted to AMSS is around three and a half months from submission to final decision. To date, AMSS has more than met the modest expectations with which it was started. It has been serving as an excellent booster for the progress in the theories and engineering applications of Solid Mechanics. However, great challenges are also brewing for the future. It will be our mission to promote mastery of these challenges in a joint effort of authors, reviewers, editors and publishers of $A M S S$.

\footnotetext{
* Corresponding author. E-mail: fangdn@pku.edu.cn
} 
This special issue of $A M S S$ commemorates the 30th Anniversary of the journal, a celebration of both the accomplishments of the past and the potential for the future. As a gift for the readers of $A M S S$, this issue is featured with a collection of 10 papers specially commissioned from among the foremost leading scientists in their fields. We sincerely hope that the international community of researchers in Solid Mechanics will appreciate the collection of papers contained in this Special Issue. Completing the process of submission, evaluation, revision, and production in time for this 30th Anniversary Issue to appear on time required the cooperation of many people, often on unreasonably tight schedules. The journal editors would like to express their appreciation not only to the distinguished contributors of the invited papers, but also to all authors, reviewers and editors who have contributed to the journal. We look forward to your continued support in the future.

Last but not least, we are particularly grateful to the Springer-Nature Publisher, who has been offering excellent cooperation in production and publication of the manuscripts in $A M S S$. 